6

OPEN ACCESS

口9⿴囗十

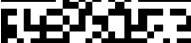

的

Open Access can to access more

free content

- Additional material is published online only. To view please visit the journal online (http://dx.doi.org/10.1136/ heartjnl-2014-307288).

${ }^{1}$ Bournemouth University, Centre of Postgraduate Medical Research \& Education, Faculty of Health and Social Sciences, UK

${ }^{2}$ Poole Hospital NHS

Foundation Trust, Dorset, UK

${ }^{3}$ Emergency Department, Royal Brisbane and Women's Hospital, Queensland, Australia ${ }^{4}$ Emergency Department, Christchurch Hospital, New Zealand

${ }^{5}$ Department of Cardiology, John Radcliffe Hospital,

Oxford, UK

${ }^{6}$ Sunshine Coast Hospital and Health Services, University of the Sunshine Coast,

Queensland, Australia

\section{Correspondence to} Dr Edward Carlton, Department of Cardiology, Poole Hospital NHS Foundation Trust, Longfleet Road, Poole, Dorset BH15 2JB, UK; eddcarlton@gmail.com

Received 4 December 2014 Revised 22 January 2015 Accepted 25 January 2015 Published Online First 17 February 2015

\section{CLlinked}

- http://dx.doi.org/10.1136/ heartjnl-2015-307555

\section{CrossMark}

To cite: Carlton EW,

Cullen $\mathrm{L}$, Than $\mathrm{M}$, et al.

Heart 2015;101:

1041-1046.

\title{
A novel diagnostic protocol to identify patients suitable for discharge after a single high-sensitivity troponin
}

\author{
Edward W Carlton, ${ }^{1,2}$ Louise Cullen, ${ }^{3}$ Martin Than, ${ }^{4}$ James Gamble, ${ }^{5}$ \\ Ahmed Khattab, ${ }^{1}$ Kim Greaves ${ }^{6}$
}

ABSTRACT

Objective To establish whether a novel accelerated diagnostic protocol (ADP) for suspected acute coronary syndrome (ACS) could successfully identify low-risk patients suitable for discharge after a single highsensitivity troponin T (hs-cTnT) taken at presentation to the emergency department. We also compared the diagnostic accuracy of this ADP with strategies using initial undetectable hs-cTnT.

Methods This prospective observational study evaluated the ability of the Triage Rule-out Using highSensitivity Troponin (TRUST) ADP to identify low-risk patients with suspected ACS. The ADP incorporated a single presentation hs-CTnT of $<14 \mathrm{ng} / \mathrm{L}$, a nonischaemic ECG and a modified Goldman risk score. Diagnostic performance of the ADP was compared with the detection limit cut-offs of hs-cTnT $(<5 \mathrm{ng} / \mathrm{L}$ and $<3 \mathrm{ng} / \mathrm{L}$ ). The primary end point was fatal/non-fatal acute myocardial infarction (AMI) within 30 days.

Results 960 participants were recruited, mean age 58.0 years, $80(8.3 \%)$ had an AMI. The TRUST ADP classified $382(39.8 \%)$ as low-risk with a sensitivity for identifying AMI of $98.8 \%$ ( $95 \% \mathrm{Cl} 92.5 \%$ to $99.9 \%$ ). hs-cTnT detection limits ( $<5 \mathrm{ng} / \mathrm{L}$ and $<3 \mathrm{ng} / \mathrm{L})$ had a sensitivity of $100 \%(94.3$ to 100$)$ and $100 \%$ (94.4 to $100)$, respectively. The TRUST ADP identified more patients suitable for early discharge at $39.8 \%$ vs $29.3 \%$ $(<5 \mathrm{ng} / \mathrm{L})$ and $7.9 \%(<3 \mathrm{ng} / \mathrm{L})(\mathrm{p}<0.001)$ with a lower false-positive rate for AMI detection; specificity $43.3 \%$ (95\% Cl $42.7 \%$ to $43.4 \%)$ vs $32.0 \%(95 \% \mathrm{Cl} 31.5 \%$ to $32.0 \%)$ and $8.6 \%(95 \% \mathrm{Cl} 8.1 \%$ to $8.6 \%)$, respectively.

Conclusions The TRUST ADP, which incorporates structured risk-assessment and a single presentation hscTnT blood draw, has potential to allow early discharge in $40 \%$ of patients with suspected ACS and has greater clinical utility than undetectable hs-cTnT strategies.

Trial registration number ISRCTN No. 21109279.

\section{INTRODUCTION}

Patients with suspected acute coronary syndrome (ACS) make up to $10 \%$ of all emergency department (ED) attendances and $25 \%$ of acute hospital admissions. ${ }^{1}$ Current guidelines recommend two serial measurements of non-high-sensitivity troponin between $6 \mathrm{~h}$ and $12 \mathrm{~h}$ after patient presentation to the ED. ${ }^{2}$ As a result, the majority of patients require prolonged assessment prior to safe discharge despite the fact that only $15-25 \%$ of these patients have a final diagnosis of ACS. ${ }^{1}$
Consensus reports suggest that high-sensitvity troponin (hs-cTn) assays may be used to reduce door-to-discharge times by using serial testing over 3-6 h. ${ }^{3}$ Investigators have reduced blood draw times further by incorporating structured clinical risk assessment protocols with hs-cTn, ${ }^{4}$ or analysing $\delta$ change over time. ${ }^{5}$ Despite successfully identifying between $40 \%$ and $60 \%$ of low-risk patients, these algorithms still require serial testing of hs-cTn which will delay discharge from the ED. This delay may be associated with significant healthcare costs, ${ }^{6}$ and contribute to ED overcrowding.

To address these issues, several studies have investigated the effectiveness of a single undetectable hs-cTn value taken at presentation to the ED in identifying those at very low risk of acute myocardial infarction (AMI) ${ }^{7-9}$ Despite demonstrating promising results as a rule-out strategy for AMI, this protocol has not been recommended by expert guidelines due to concerns over assay analytical interference and poor test specificity. ${ }^{3}$ Therefore, a clinically applicable protocol that allows the discharge of a significant proportion of patients after just a single hs-cTn blood draw at presentation remains an attractive yet elusive goal.

Using binary hs-cTn results alone to guide discharge decisions fails to use a wealth of clinical information available to treating physicians. The Goldman risk score ${ }^{10}$ uses simple variables that are immediately available to the ED physician and are derived from the history, examination and ECG findings. Since its inception, the score has been modified to improve physician decision making in the identification of low-risk patients. This has led to improved use of hospital resources. ${ }^{11}$ Despite achieving the highest level of evidentiary support for use in ED patients with chest pain the modified Goldman (m-Goldman) risk score remains untested as a discharge tool in combination with a single presentation hs-cTn.

The Triage Rule-out Using high-Sensitivity Troponin (TRUST) study's primary aim was to establish whether a novel accelerated diagnostic protocol (ADP) for patients with suspected ACS consisting of hs-cTn, a non-ischaemic ECG and the m-Goldman score, could successfully identify low-risk patients suitable for discharge after a single blood draw at presentation to the ED. Secondary aims were to compare the diagnostic accuracy of the ADP with strategies using initial undetectable hs-cTnT levels. 


\section{METHODS}

This prospective observational clinical trial was designed to assess the predefined TRUST ADP. The protocol was designed to be truly pragmatic in order to enhance the widespread applicability of the study results ${ }^{12}$; with attending clinicians performing m-Goldman risk scores, rostered clinical (not research) staff undertaking blood sampling, real-time sample processing and 24/7 recruitment. The study was designed using the Standards for Reporting Diagnostic Accuracy. ${ }^{13}$ and approved by the UK National Research Ethics Service. All participants provided written informed consent. The TRUST study was registered with the Controlled Trials Database (ISRCTN No. 21109279) and complies with the Declaration of Helsinki.

\section{Study setting, recruitment and data collection}

Poole NHS Foundation Trust is a UK District General Hospital, its ED has approximately 62000 new patient attendances per year. Patients with suspected ACS are managed according to the local hospital protocol, which involves risk assessment by ED physician staff using the m-Goldman risk score and blood drawn for hs-cTnT at $6 \mathrm{~h}$ after presentation. As part of the study protocol, blood was also taken at presentation for hs-cTnT analysis. While historical clinical protocols, at the time of this study, did not include troponin measurement at presentation, this had the benefit of ensuring that treating physicians were blinded to the initial hs-cTnT result to avoid selection bias. ${ }^{14}$

The fifth generation Roche ELECSYS hs-cTnT assay (Roche, Switzerland), which has a limit of detection (lowest analyte concentration likely to be reliably distinguished from the limit of blank at which detection is feasible) of $5 \mathrm{ng} / \mathrm{L}$, limit of blank (highest apparent analyte concentration expected to be found when replicates of a blank sample containing no analyte are tested) of $3 \mathrm{ng} / \mathrm{L}$, 99th centile of $14 \mathrm{ng} / \mathrm{L}$ and $10 \%$ coefficient of variation of $<10 \%$ at $9 \mathrm{ng} / \mathrm{L}$, was used for research (presentation) and reference (6-h) samples. During initial assessment clinical staff drew blood for routine admission samples and an additional $3.5 \mathrm{~mL}$ of whole blood in a prelabelled study-specific serum settling tube for hs-cTnT analysis. All serum samples were tested in real time.

Consecutive patients attending the ED with suspected ACS were prospectively screened from July 2012 to August 2013. Patients were included if they were at least 18 years of age and had at least $5 \mathrm{~min}$ of chest pain suggestive of ACS, and for whom the attending physician determined inpatient evaluation was required. Possible cardiac symptoms included acute chest, epigastric, neck, jaw or arm pain, or discomfort or pressure without an apparent non-cardiac source, in accordance with the American Heart Association case definitions. ${ }^{15}$ Patients were excluded if any of the following were present: ST-segment elevation myocardial infarction or left bundle branch block not known to be old, ECG changes diagnostic of ischaemia (ST segment depression $\geq 1 \mathrm{~mm}$ or T-wave inversion consistent with the presence of ischaemia), ${ }^{2}$ arrhythmias (new-onset atrial fibrillation, atrial flutter, sustained supraventricular tachycardia, second-degree or complete heart block, or sustained or recurrent ventricular arrhythmias), hs-cTnT not suitable for analysis (eg, haemolysis), age $\geq 80$ years, atypical symptoms in the absence of chest discomfort, a clear non-ACS cause for chest pain was found at presentation (eg, pulmonary embolism, pneumonia, aortic dissection), another medical condition requiring hospital admission, refusal or inability to give informed consent, non-English speaking, pregnancy, renal failure requiring dialysis or inability to be contacted after discharge.

Data were collected prospectively using a published data dictionary. ${ }^{16}$ Attending ED clinicians completed the m-Goldman risk score on a predesigned clinical report form. Follow-up was undertaken by independent review of hospital electronic patient records, summary of health records from the patient's general practitioner (GP) obtained at least 6 months after attendance and a national clinical records search (which identifies death). The ethics committee did not grant permission for direct patient contact as they felt that comprehensive follow-up data relating to adverse events could be obtained accurately through GP records. This is because in the UK, GPs hold comprehensive records for individuals relating to primary, secondary and tertiary care. GP records have been demonstrated to be more accurate at reporting hospital admissions, including those for cardiac related events, than patients. ${ }^{17}$ GPs were therefore requested to provide all information regarding presentation to other institutions with chest pain, cardiology outpatient review and cardiac testing, including angiography with or without intervention. Where a participant had not attended hospital follow-up and/or a GP had failed to provide a health record/not GP-registered, the patient was regarded as lost to follow-up.

\section{Index tests}

The primary index test was the TRUST ADP (table 1), this defined a patient as 'low-risk' if all of the following conditions were satisfied at presentation: a m-Goldman Score of 0 or 1, a non-ischaemic ECG and a single central laboratory hs-cTnT of $<14 \mathrm{ng} / \mathrm{L}$ at presentation.

Secondary index tests were the detection limits for hs-cTnT $(5 \mathrm{ng} / \mathrm{L}$ and $3 \mathrm{ng} / \mathrm{L}$ ) and non-ischaemic ECG at presentation.

\section{Outcome measures}

The primary end point was the presence of fatal or non-fatal AMI occurring within 30 days of hospital attendance (including the index visit).

The presence of AMI was defined according to the Third Universal Definition of MI which states that a rise and/or fall in

Table 1 The Modified Goldman Score and the TRUST accelerated diagnostic protocol (ADP)

Modified Goldman risk score

1 point for each variable present

Typical new-onset chest pain at rest

Pain the same as previous myocardial

infarction

Pain not relieved by glyceryl trinitrate

(GTN) spray within $15 \mathrm{~min}$

Pain lasting more than $60 \mathrm{~min}$

Pain occurring with increasing frequency

Hypotension (systolic blood pressure

$<100 \mathrm{~mm} \mathrm{Hg}$ )

Acute shortness of breath

Pain within 6 weeks of a myocardial

infarction or revascularisation

Modified Goldman total

Trust ADP

Low risk* (Suitable for discharge)

1. Modified Goldman score $\leq 1$

2. Non-ischaemic ECG

3. Presentation high-sensitivity troponin $\mathrm{T}<14 \mathrm{ng} / \mathrm{L}$

Not low risk

1. Modified Goldman score $>1$

2. Ischaemic ECG

3. Presentation high-sensitivity troponin $\mathrm{T} \geq 14 \mathrm{ng} / \mathrm{L}$

*Safety point: protocol not validated in age $\geq 80$ years.

TRUST, Triage Rule-out Using high-Sensitivity Troponin. 


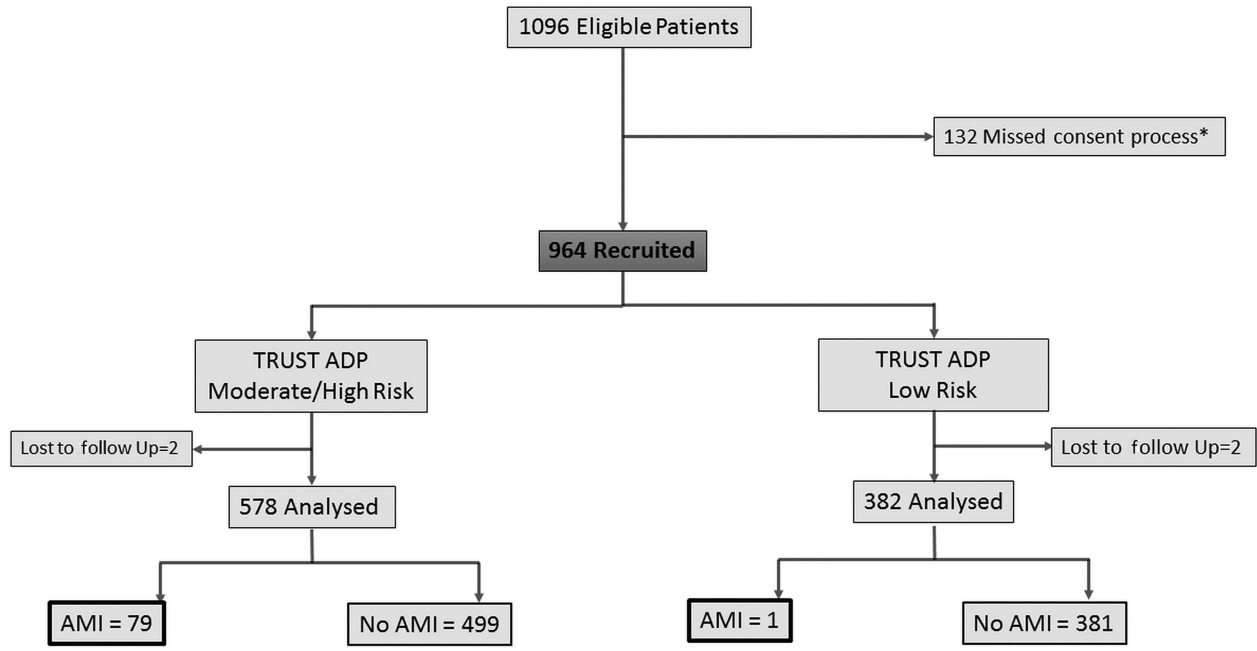

Figure 1 Participant recruitment flow chart. The 132 patients who missed the consent process were similar in age, gender, risk factors and m-Goldman scores ( $p>0.05$ for all). ADP, accelerated diagnostic protocol; AMI, acute myocardial infarction; TRUST, Triage Rule-out Using high-Sensitivity Troponin.

troponin, with at least one value above the 99th centile value in the context of a patient with ischaemic symptoms or signs (ECG changes or imaging evidence) would satisfy the diagnosis. ${ }^{18}$ Based on current consensus guidance for hs-cTn assays, a rise or fall of $20 \%(\delta)$ was considered statistically significant and consistent with a diagnosis of $\mathrm{AMI}{ }^{3}$ Adjudication of the primary end point was carried out by two local cardiologists blinded to the m-Goldman score but who had access to the clinical record, ECG and serial hs-cTnT results. If a troponin result was above the 99th centile value and a non-ischaemic cause of troponin elevation was identified this was considered by the adjudicating cardiologist in accordance with expert consensus. ${ }^{19}$

The presence of major adverse cardiac events (MACEs) occurring within 30 days of hospital attendance (including the index visit) was a secondary outcome measure. MACE included: death due to ischaemic heart disease, cardiac arrest, urgent revascularisation, cardiogenic shock, ventricular arrhythmia, high-degree atrioventricular block needing intervention and AMI. MACE was defined according to previous large scale studies assessing the safety of rapid discharge protocols. 42021

Table 2 Patient characteristics

\begin{tabular}{|c|c|c|c|c|}
\hline & Total $(\mathrm{N}=960)$ & $\begin{array}{l}\text { Fatal/non-fatal AMI } \\
\text { positive at } 30 \text { days }(\mathrm{N}=80)\end{array}$ & $\begin{array}{l}\text { TRUST ADP intermediate/high } \\
\text { risk ( } N=578)\end{array}$ & $\begin{array}{l}\text { TRUST ADP low risk } \\
(\mathrm{N}=382)\end{array}$ \\
\hline Age, years $($ Mean \pm SD) & $58.0 \pm 13.3$ & $63.3 \pm 10.6$ & $60.4 \pm 12.8$ & $55.6 \pm 19.4$ \\
\hline Sex (\% male) & $565(58.9)$ & $53(66.3)$ & $360(62.3)$ & $205(53.7)$ \\
\hline Ethnicity (\% British Caucasian) & $914(95.2)$ & $72(90.0)$ & $549(95.0)$ & $365(95.5)$ \\
\hline \multicolumn{5}{|l|}{ Risk factors N (\%) } \\
\hline Hypertension & $452(47.1)$ & $59(73.8)$ & $319(55.2)$ & $123(34.8)$ \\
\hline Diabetes & 164 (17.1) & $20(25.0)$ & $124(21.4)$ & $40(10.5)$ \\
\hline Dyslipidaemia & $635(66.1)$ & $63(78.6)$ & $429(74.2)$ & $206(53.9)$ \\
\hline Smoking current & $231(24.1)$ & $19(23.8)$ & $129(22.3)$ & $102(26.7)$ \\
\hline Smoker ex & $343(35.1)$ & $30(37.5)$ & 229 (39.6) & $114(29.8)$ \\
\hline Family history of coronary artery disease & $354(36.9)$ & $29(36.3)$ & $215(37.2)$ & $139(36.4)$ \\
\hline \multicolumn{5}{|l|}{ Medical history } \\
\hline Angina & $251(26.1)$ & $29(36.3)$ & $207(35.8)$ & $44(11.5)$ \\
\hline Myocardial infarction & $204(21.3)$ & $26(32.5)$ & $174(30.1)$ & $30(7.9)$ \\
\hline Percutaneous coronary intervention & $183(19.1)$ & $22(27.5)$ & $146(25.3)$ & $37(9.7)$ \\
\hline Congestive cardiac failure & $30(3.1)$ & $4(5.0)$ & $25(4.3)$ & $5(1.3)$ \\
\hline Atrial arrhythmia & $119(12.4)$ & $8(10.0)$ & $86(14.9)$ & $33(8.6)$ \\
\hline Stroke & $63(6.6)$ & $5(6.3)$ & $45(7.7)$ & $18(4.7)$ \\
\hline Coronary artery bypass graft & $50(5.2)$ & $7(8.8)$ & $41(7.1)$ & $9(2.4)$ \\
\hline \multicolumn{5}{|l|}{ Baseline medications } \\
\hline Aspirin & 361 (37.6) & $40(50.0)$ & $276(47.8)$ & $85(22.3)$ \\
\hline Clopidogrel & $112(11.7)$ & $8(10.0)$ & $84(14.5)$ & $28(7.3)$ \\
\hline$\beta$ blocker & $281(29.3)$ & $25(31.3)$ & $210(36.3)$ & $71(18.6)$ \\
\hline ACE inhibitor & $272(28.3)$ & $29(36.3)$ & 195 (33.7) & $77(20.2)$ \\
\hline Statin & $369(38.4)$ & $37(46.3)$ & $276(47.8)$ & $93(24.3)$ \\
\hline Median length of hospital stay $(\mathrm{h}) \pm \mathrm{QQR}$ & $18.8 \pm 32.4$ & $107.5 \pm 110.3$ & $22.4 \pm 62.0$ & $14.0 \pm 11.9$ \\
\hline
\end{tabular}




\section{Statistical analysis}

Baseline characteristics of the study population were analysed with conventional group descriptive statistics. Diagnostic protocol results and outcome status were cross-tabulated to permit calculation of sensitivity, specificity, negative predictive value (NPV), positive predictive value, positive likelihood ratio and negative likelihood ratio. Statistical significance was evaluated using McNemar's test. All statistical analysis was carried out using SPSS V.20.

\section{RESULTS}

Nine hundred and sixty-four consenting patients were recruited (figure 1), Four patients were lost to follow-up (health records pertaining to presence of outcome measures unobtainable) meaning that $99.6 \%$ were successfully monitored for 30 days. However, no patient lost to follow-up died within 30 days of attendance. Participants were predominantly white, older men who commonly had risk factors for coronary artery disease (table 2). Of the patients $80 / 960(8.3 \%)$ had a primary outcome event (fatal or non-fatal AMI) and 97/960 (10.1\%) had a MACE within 30 days, and 30/960 (3.1\%) patients had a nonischaemic cause of hs-cTnT elevation above the 99th centile identified (diagnoses summarised in the online supplementary appendix). Patients presented to the ED at a median of $2 \mathrm{~h}$ $20 \mathrm{~min}(\mathrm{IQR} \pm 228 \mathrm{~min}$ ) after chest pain onset. Blood was taken for hs-cTnT at a median of $35 \mathrm{~min}(\mathrm{IQR} \pm 14 \mathrm{~min}$ ) after patient arrival.

\section{Diagnostic accuracy of the TRUST ADP}

The TRUST ADP classified 382/960 (39.8\%) of patients as at low risk of fatal or non-fatal AMI (table 3), with a sensitivity for identifying AMI of 98.8\% (95\% CI 92.4\% to $99.9 \%$ ) and NPV of $99.7 \%$ (95\% CI $98.4 \%$ to $100 \%)$ and had a similar diagnostic performance for the secondary outcome measure (MACE) (table 4).

A single patient $(0.3 \%)$ classified as low-risk by the TRUST ADP had an AMI during the initial hospital attendance and follow-up. This patient was a 78-year-old woman classified as low-risk on the m-Goldman score and had a hs-cTnT of $13 \mathrm{ng} / \mathrm{L}$ at presentation. However, a minor hs-cTnT elevation to $20 \mathrm{ng} / \mathrm{L}$ ( $\delta$ change 27\%) occurred on the second hs-cTnT test at $6 \mathrm{~h}$ and was therefore diagnosed with an AMI. The patient was medically managed and had no further complications.

\section{Undetectable troponin strategies}

The diagnostic performance of hs-cTnT limit of detection cut-off values in patients with a non-ischaemic ECG are shown in table 4. By using the limit of detection cut-off value of $5 \mathrm{ng} / \mathrm{L}$ for the primary outcome measure (AMI) the sensitivity was $100 \%$ (95\% CI $94.3 \%$ to $100 \%)$ and $270 / 922(29.3 \%)$ of patients were eligible for early discharge (table 3). However, using the secondary outcome measure (MACE), three patients (1.1\%) identified as suitable for discharge using this strategy required urgent revascularisation (all three were aged in their 40 s, two had severe left anterior descending artery disease and one severe right coronary artery disease). Using the limit of blank $(<3 \mathrm{ng} / \mathrm{L})$ the sensitivity for fatal/non-fatal AMI was $100 \%$ (95\% CI $94.4 \%$ to $100 \%$ ) and only $7.9 \%$ would have been eligible for early discharge. One patient $(1.4 \%)$ with a hs-cTnT $<3 \mathrm{ng} / \mathrm{L}$ required urgent revascularisation.
Table 3 Occurrence of fatal/non-fatal AMI and MACE during the index hospital visit or at 30 days according to index test

\begin{tabular}{|c|c|c|c|}
\hline & AMI & No AMI & Total \\
\hline \multicolumn{4}{|l|}{ TRUST ADP } \\
\hline Not low risk & 79 & 499 & 578 \\
\hline Low risk & 1 & 381 & 382 \\
\hline \multicolumn{4}{|l|}{ Hs-cTnT $<5 \mathrm{ng} / \mathrm{L}^{*}$} \\
\hline$\geq 5 \mathrm{ng} / \mathrm{L}$ & 78 & 574 & 652 \\
\hline$<5 \mathrm{ng} / \mathrm{L}$ & 0 & 270 & 270 \\
\hline \multicolumn{4}{|l|}{ Hs-cTnT $<3$ ng/L ${ }^{*}$} \\
\hline$\geq 3 \mathrm{ng} / \mathrm{L}$ & 78 & 771 & 849 \\
\hline \multirow[t]{2}{*}{$<3 \mathrm{ng} / \mathrm{L}$} & 0 & 73 & 73 \\
\hline & MACE & No MACE & Total \\
\hline \multicolumn{4}{|l|}{ TRUST ADP } \\
\hline Not low risk & 96 & 482 & 578 \\
\hline Low risk & 1 & 381 & 382 \\
\hline \multicolumn{4}{|l|}{ Hs-cTnT $<5 \mathrm{ng} / \mathrm{L}^{*}$} \\
\hline$\geq 5 \mathrm{ng} / \mathrm{L}$ & 92 & 560 & 652 \\
\hline$<5 \mathrm{ng} / \mathrm{L}$ & 3 & 267 & 270 \\
\hline \multicolumn{4}{|l|}{$\mathrm{Hs}-\mathrm{cTnT}<3 \mathrm{ng} / \mathrm{L}^{*}$} \\
\hline$\geq 3 \mathrm{ng} / \mathrm{L}$ & 94 & 755 & 841 \\
\hline$<3 \mathrm{ng} / \mathrm{L}$ & 1 & 72 & 73 \\
\hline
\end{tabular}

\section{Comparison of strategies}

The TRUST ADP identified significantly more patients suitable for immediate discharge at $39.8 \%$ vs $29.3 \%(<5 \mathrm{ng} / \mathrm{L})$ and $7.9 \%(<3 \mathrm{ng} / \mathrm{L})(\mathrm{p}<0.001)$ with a lower false-positive rate for AMI detection; specificity $43.3 \%$ (95\% CI $42.7 \%$ to $43.4 \%$ ) vs $32.0 \%$ (95\% CI $31.5 \%$ to $32.0 \%$ ) and $8.6 \%$ (95\% CI $8.1 \%$ to $8.6 \%)$ respectively, while maintaining a high diagnostic accuracy for the rule-out of AMI.

\section{DISCUSSION}

This study demonstrates that the TRUST ADP for suspected ACS can successfully identify $40 \%$ of patients as low-risk after just a single hs-cTnT taken at presentation to the ED, with a NPV of $>99.5 \%$. When compared with strategies using undetectable hs-cTnT, more patients are eligible for early discharge with lower false-positive rates, suggesting this approach has greater clinical utility. Furthermore, by incorporating clinical risk stratification, the TRUST ADP has improved accuracy in identifying those who require urgent revascularisation.

Our results suggest that the introduction of this ADP has the potential to reduce the length of stay for low-risk patients (currently $14 \mathrm{~h}$ in our institution) after a single laboratory-based troponin and avoid the necessity for two separate blood draws. Uptake of this protocol may have significant benefits for healthcare services worldwide by reducing hospital admission rates, ED overcrowding, duplication of staff time and resource use. Furthermore, by using ED physicians to carry out riskstratification and real-time troponin sampling with 24-h recruitment we have demonstrated that this ADP is truly applicable.

This analysis confirms the results of recent large-scale exploratory research that showed undetectable hs-cTnT held promise as a tool for rule-out of AMI or death. ${ }^{9}$ However, we demonstrate that by using MACE (which also includes urgent revascularisation) missed-event rates of the undetectable troponin strategies 
rise above $1 \%$, this may be unacceptable to the majority of ED clinicians. $^{22}$ Therefore, consistent with consensus guidelines, ${ }^{3}$ we cannot recommend uptake of undetectable hs-cTnT rule-out strategies in this setting.

Our data suggest that focus move away from strategies that use a stand-alone single initial undetectable hs-cTn result to guide discharge decisions, and towards protocols that also incorporate structured clinical risk assessment. A number of reports combining these two strategies have been reported recently and show early promise. For example, the History, ECG, Age, Risk Factors and Troponin (HEART) score, ${ }^{23}$ may enable safe early discharge after a single troponin at presentation but requires validation with hs-cTn, and the Manchester Acute Coronary Syndromes (MACS) decision rule, ${ }^{24}$ has demonstrated excellent discriminatory power but requires the use of hearttype fatty acid binding protein in addition to hs-cTn. Prospective comparison of these strategies is required.

There are some limitations to this study. The inclusion of predominantly British Caucasian patients may limit the applicability to international settings. The upper age cut-off of $\geq 80$ years was chosen for pragmatic reasons. In our institution, patients above this age are admitted to a separate and dedicated assessment area. Therefore we recognise that this may affect the applicability of TRUST ADP in those $>80$ years of age.

Patients were only recruited if they had a non-ischaemic ECG at presentation-thereby reducing the prevalence of MACE in the study population. Expansion of the inclusion criteria to include those patients with ECG changes consistent with ACS would have added little practical value because this group is not suitable for early discharge anyway. We therefore intentionally excluded patients with a clear diagnosis of ACS to focus on a particular group that remains a major diagnostic challenge.

\section{Key messages}

What is already known on this subject?

The use of undetectable high-sensitivity troponin levels and risk scores in combination with early biomarker testing have recently been put forward as diagnostic tools aiming to reduce door-to-discharge times in patients with suspected acute coronary syndromes. However, a clinically applicable protocol that allows the discharge of a significant proportion of patients after just a single high sensitivity troponin blood draw at presentation to the emergency department remains an attractive yet elusive goal.

\section{What might this study add?}

Using a simple clinical risk score, together with the results of a single high-sensitivity troponin result, the Triage Rule-out Using high-Sensitivity Troponin accelerated diagnostic protocol, may enable immediate discharge in up to $40 \%$ of patients. This strategy identifies more patients suitable for early discharge, with lower false-positive rates than undetectable troponin strategies.

\section{How might this impact on clinical practice?}

Chest pain makes up a quarter of medical admissions in the UK. A diagnostic strategy that prevents unnecessary hospital admission in a large proportion of this patient group would have significant benefits for healthcare services by reducing hospital admission rates, emergency department overcrowding, duplication of staff time and resource use. 
We recognise that the TRUST ADP now requires validation as part of a multicentre randomised controlled trial. However, without first analysing the safety of this diagnostic strategy through an observational cohort design, the principle of clinical equipoise may not have justified a randomised study design. ${ }^{25}$

\section{CONCLUSION}

The TRUST ADP, which incorporates a structured riskassessment and single presentation hs-cTnT blood draw, has the potential to allow early discharge in $40 \%$ of patients with suspected ACS. This ADP has superior clinical utility when compared with undetectable hs-cTnT strategies. Future research should focus on methodologies that incorporate clinical assessment with hs-cTn testing rather than troponin testing alone.

\section{Twitter Follow Edward Carlton at @eddcarlton}

Acknowledgements The authors thank Dr John Beavis (Bournemouth University) for statistical support, and Georgina Gemmell, Dr Elena Cowan and staff at Poole Hospital emergency department and Biochemistry departments for their assistance and support. The authors also thank Dr Nick Jenkins (Emergency Department, Wexham Park Hospital) for assistance with concept development

Contributors Each author has contributed to the analysis and interpretation of the data, and drafting and approval of the final manuscript. All authors have also contributed to the conception/design of the study reported in this manuscript.

Funding The TRUST Study was supported by a research grant from the Royal College of Emergency Medicine of the UK and research fellowship funding from Bournemouth University, UK.

Competing interests EWC has received funding from Abbott in support for related research. LC has received funding from Abbott, Roche, Alere, Siemens and Radiometer Pacific for clinical trials, and from Alere, Boehringer Ingelheim, Pfizer, Astra Zeneca, Abbott, Novartis and Radiometer Pacific for speaking and education. MT has received funding from Alere, Abbott, Beckman and Roche for speaking and support for other research. KG has received funding from AstraZeneca and Takeda UK for related research.

Patient consent Obtained.

Ethics approval Frenchay Research Ethics Committee (reference 12/SW/0133).

Provenance and peer review Not commissioned; externally peer reviewed.

Data sharing statement All requests for further data from this study should be addressed to the corresponding author.

Open Access This is an Open Access article distributed in accordance with the Creative Commons Attribution Non Commercial (CC BY-NC 4.0) license, which permits others to distribute, remix, adapt, build upon this work non-commercially, and license their derivative works on different terms, provided the original work is properly cited and the use is non-commercial. See: http://creativecommons.org/ licenses/by-nc/4.0/

\section{REFERENCES}

1 Goodacre S, Cross E, Arnold J, et al. The health care burden of acute chest pain. Heart 2005:91:229-30.

2 Amsterdam E, Kirk J, Bluemke D, et al. American Heart Association Exercise, Cardiac Rehabilitation, and Prevention Committee of the Council on Clinical Cardiology, Council on Cardiovascular Nursing, and Interdisciplinary Council on Quality of Care and Outcomes Research. Testing of low risk patients presenting to the emergency department with chest pain: a scientific statement from the American Heart Association. Circulation 2010;122:1756-76.

3 Thygesen K, Mair J, Giannitsis E, et al.; Study Group on Biomarkers in Cardiology of ESC Working Group on Acute Cardiac Care. How to use high-sensitivity cardiac troponins in acute cardiac care. Eur Heart J 2012:33:2252-7.
4 Cullen L, Mueller C, Parsonage W, et al. Validation of high-sensitivity troponin I in a 2-hour diagnostic strategy to assess 30-day outcomes in emergency department patients with possible acute coronary syndrome. J Am Coll Cardiol 2013:62:1242-9.

5 Reichlin T, Schindler C, Drexler B, et al. One-Hour Rule-out and Rule-in of Acute Myocardial Infarction Using High-Sensitivity Cardiac Troponin T. Arch Intern Med 2012;172:1211-8.

6 Thokala P, Goodacre S, Collinson P, et al. Cost-effectiveness of presentation versus delayed troponin testing for acute myocardial infarction. Heart 2012;98:1498-503.

7 Body R, Carley S, McDowell G, et al. Rapid exclusion of acute myocardial infarction in patients with undetectable troponin using a high-sensitivity assay. J Am Coll Cardiol 2011:58:1332-9.

8 Rubini Giminez M, Hoeller R, Reichlin T, et al. Rapid rule out of acute myocardial infarction using undetectable levels of high-sensitivity cardiac troponin. Int I Cardiol 2013;168:3896-901.

9 Bandstein $\mathrm{N}$, Ljung $\mathrm{R}$, Johansson $\mathrm{M}$, et al. Undetectable high sensitivity cardiac troponin $\mathrm{T}$ level in the Emergency Department and risk of Myocardial Infarction. J Am Coll Cardiol 2014;63:2569-78.

10 Goldman L, Cook E, Johnson $P$, et al. Prediction of the need for intensive care in patients who come to the emergency departments with acute chest pain. $N$ Engl J Med 1996:334:1498-504

11 Reilly B, Evans A, Schaider J, et al. Impact of a clinical decision rule on hospital triage of patients with suspected acute cardiac ischemia in the emergency department. JAMA 2002;288:342-50

12 Tunis S, Stryer D, Clancy C. Practical clinical trials: increasing the value of clinical research for decision making in clinical and health policy. JAMA 2003;290:1624-32.

13 Bossuyt $\mathrm{P}$, Reitsma JB, Bruns D, et al. Standards for Reporting of Diagnostic Accuracy. The STARD statement for reporting studies of diagnostic accuracy: explanation and elaboration. Ann Intern Med 2003;138:W1-12.

14 Stiell I, Wells G. Methodologic standards for the development of clinical decision rules in emergency medicine. Ann Emerg Med 1999;33:437-47.

15 Luepker R, Apple F, Christenson R, et al. Case definitions for acute coronary hear disease in epidemiology and clinical research studies: a statement from the AHA Council on Epidemiology and Prevention; AHA Statistics Committee; World Heart Federation Council on Epidemiology and Prevention; Centers for Disease Control and Prevention; and the National Heart, Lung, and Blood Institute. Circulation 2003; 108:2543-9.

16 Cullen L, Than M, Brown A, et al. Comprehensive standardized data definitions for acute coronary syndrome research in emergency departments in Australasia. Emerg Med Australas 2010;22:35-55

17 Taylor F, Reeves B, Ascione $\mathrm{R}$, et al. Accuracy of reporting cardiac related events during long term follow up. Heart 2003:89:331-2.

18 Thygesen $\mathrm{K}$, Alpert J, Jaffe A, et al. Third universal definition of myocardial infarction. J Am Coll Cardiol 2012;60:1581-98

19 Newby L, Jesse R, Babb J, et al. ACCF 2012 expert consensus document on practical clinical considerations in the interpretation of troponin elevations: a report of the American College of Cardiology Foundation task force on Clinical Expert Consensus Documents. J Am Coll Cardiol 2012;60:2427-63.

20 Goodacre S, Bradburn M, Cross E, et al.; RATPAC Research Team. The Randomised Assessment of Treatment using Panel Assay of Cardiac Markers (RATPAC) trial: a randomised controlled trial of point-of-care cardiac markers in the emergency department. Heart 2011;97:190-6.

21 Than M, Cullen L, Reid CM, et al. A 2-h diagnostic protocol to assess patients with chest pain symptoms in the Asia-Pacific region (ASPECT): a prospective observational validation study. Lancet 2011;377:1077-84.

22 Than $M$, Herbert $M$, Flaws $D$, et al. What is an acceptable risk of major adverse cardiac events in chest pain patients soon after discharge from the Emergency Department?: a clinical survey. Int J Cardiol 2012;166:752-4

23 Backus B, Six A, Kelder J, et al. A prospective validation of the HEART score for chest pain patients at the emergency department. Int J Cardiol 2013;168:2153-8.

24 Body R, Carley S, Mcdowell G, et al. The Manchester Acute Coronary Syndromes (MACS) decision rule for suspected cardiac chest pain: derivation and external validation. Heart 2014;100:1462-8.

25 Freedman B. Equipoise and the ethics of clinical research. $N$ Eng/ J Med $1987 ; 317: 141-5$ 